\title{
RECOGNIZING COMMERCIALS IN REAL-TIME USING THREE VISUAL DESCRIPTORS AND A DECISION-TREE
}

\author{
Ronald Glasberg, Cengiz Tas and Thomas Sikora \\ glasberg@nue.tu-berlin.de,ctas@cs.tu-berlin.de,sikora@nue.tu-berlin.de \\ Communication Systems Group \\ Technical University Berlin \\ 10587 Berlin, Germany
}

\begin{abstract}
We present a new approach for classifying mpeg-2 video sequences as 'commercial' or 'non-commercial' by analyzing specific color, texture and motion features of consecutive frames in real-time. This is part of the wellknown video-genre-classification problem, where popular TV-broadcast genres like cartoon, commercial, music, news and sports are studied. Such applications have also been discussed in the context of MPEG-7 [11]. In our method the extracted features from three visual descriptors are logically combined using a decision tree to produce a reliable recognition. The results demonstrate a high identification rate based on a large collection of 200 representative video sequences (40 'commercials' and 4*40 'non-commercials') gathered from free digital TV-broadcasting in Germany.
\end{abstract}

\section{INTRODUCTION}

With the advent of digital TV-broadcasts presenting more than hundred of channels at a time, the need for a userfriendly TV-program selection is growing. Unlike the present TV, a new system should enable users to access programs clustered by genres.

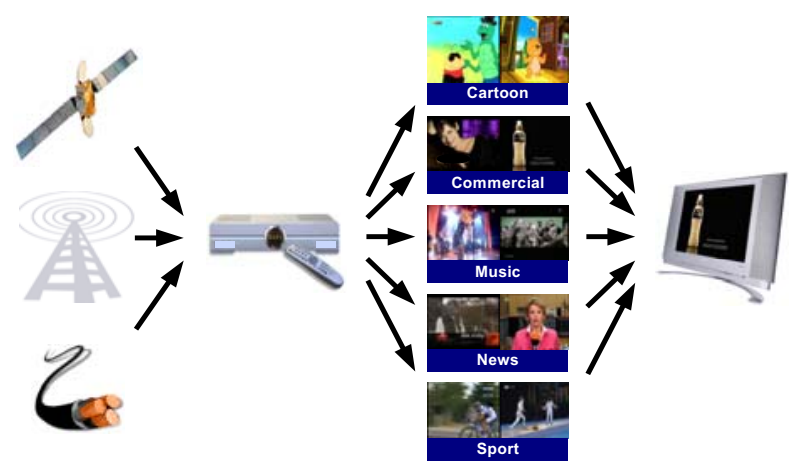

Figure 1: Concept of a Video-Genre-Classification System

Our interest is the feature-based detection of commercials in broadcasting TV material.

\section{RELATED WORK}

The recent approaches addressing commercial-detection and video-classification are listed in [1-6] and [7-9] respectively. Satterwhite et al. describe [2] the characteristics of commercials and give an overview of several algorithms, which have been experimentally used for detection:

1. Appearance of several monochrome black frames between each commercial block. In this context Lienhart et al. published an approach [6], requiring that the average and the standard deviation intensity values of the pixels in these frames should be below a certain threshold. Sadlier et al. [5] designed a method to detect black frames using the DCcoefficients in an MPEG-1-encoded bit stream.

We extend these methods in order to prevent the false acceptance of dark frames belonging to fades within a commercial spot, but still showing a small area of information.
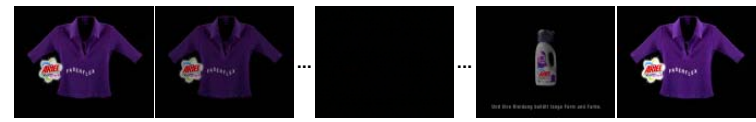

Figure 2: Candidate frames for false acceptance as a separating block being part of a fade

Therefore we developed a descriptor, which analyses subimages of a frame and the time-distance between the blocks.

2. Removal of the TV-logo during the commercial blocks. The recognition of logos is described in [1, 4]. These methods are computationally expensive and therefore not suitable for our real-time application. We introduce a fast descriptor, which detects the presence of a transparent or non-transparent logo.

3. Combining the features with a complex classifier. We developed three visual descriptors and combined them with a decision tree according to the ID3 algorithm [10]. The main goal of our research is the classification of an mpeg-2 video-stream in real-time into genres at the highest level [7]. 


\section{COMMERCIAL-CLASSIFICATION PROCESS}

In a first step the visual data is extracted from the mpeg-2 streams.

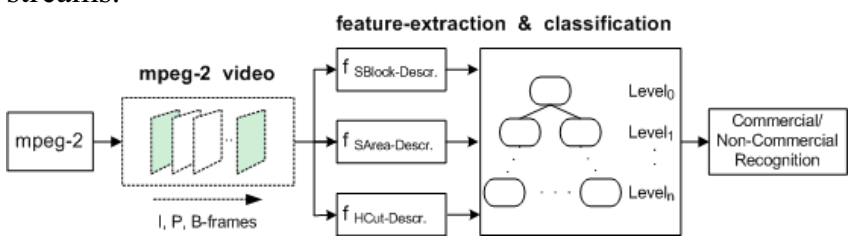

Figure 3: Process involved computing the feature vectors

We consider all consecutive frames of the image sequence and save them temporarily for processing.

\subsection{Visual-Descriptors}

German commercials have several characteristic features in common. They are shown in Figure 4, like:

(a) appearance of 3 to 14 separating frames between each commercial block,

(b) duration of individual commercials more than 10 and less than 60 seconds,

(c) removal of the network-logo during the commercial breaks and

(d) high number of cuts within the spots.

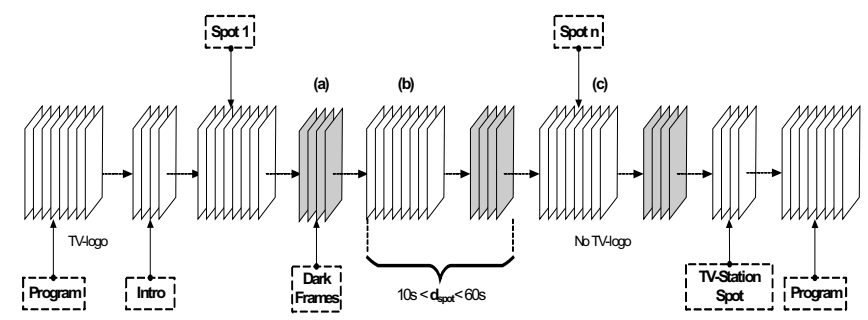

Figure 4: Structure of a commercial block

Based on these observations, we designed three descriptors to transform our data into feature vectors.

\subsubsection{Separating-Block Descriptor}

The descriptor is depicted in Figure 5. We determine the average brightness $L_{\mu}$ as well as the variance $L_{v a r}$ of the pixels for each frame. All candidates as separating frames have to be below a certain threshold. Next we examine the average brightness $L_{\mu-s b}$ for $3 \times 3$ sub-images of the selected frame. The number of consecutive frames, satisfying these requirements, is counted and has to be in a certain range (a). This is called a separating block $f_{l}$, if the time distance to the previous separating block fulfills the restrictions (b) regarding the duration of individual commercials.

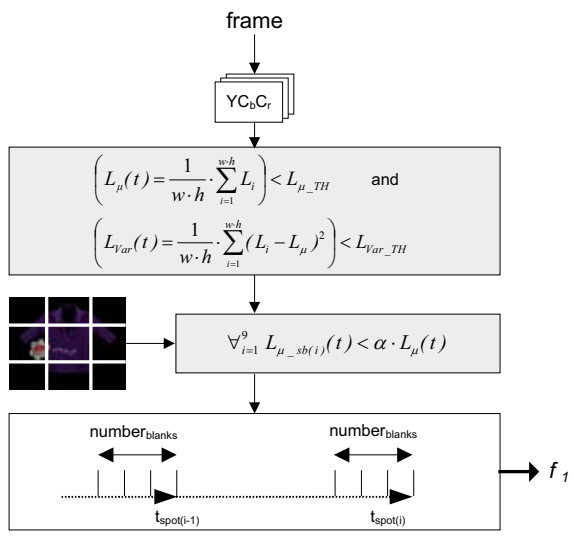

Figure 5: Block diagram of the separating-block descriptor

\subsubsection{Static-Area Descriptor}

The descriptor is depicted in Fig. 6. We scan the areas $c_{0^{-}} c_{3}$, where a TV-logo is displayed usually. The corresponding brightness values of the first frame of a processing window $\mathrm{N}$ are saved in $L_{b u f f e r}$. For each consecutive frame, we compare the current values $L_{\text {actFrame }}$ with $L_{\text {buffer }}$ and save the darkest values of the search areas. After a length of $\mathrm{N}$ frames, a binary image is generated by comparing the resulting values to a threshold. If the average brightness value $\mu_{c}$ of at least one of the four corners is higher than zero, we detected static pixels $f_{2}$ and the probability of a logo, present in this area, is high.

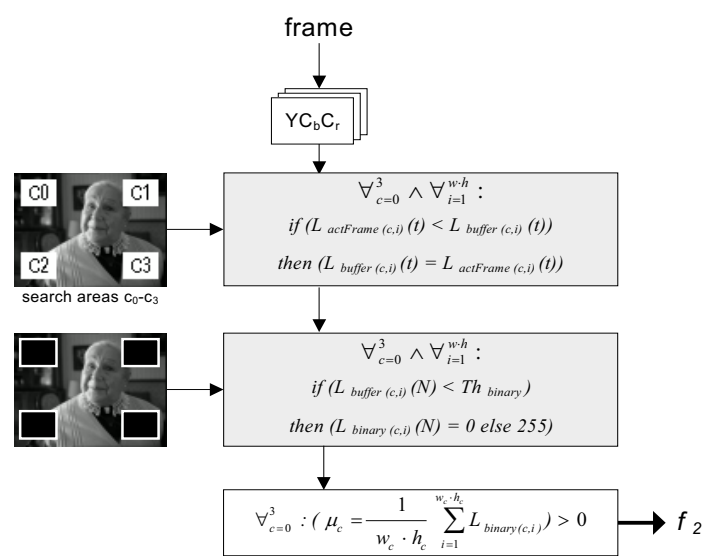

Figure 6: Block diagram of the static-area descriptor

\subsubsection{Hard-Cut Descriptor}

The difference of two consecutive I-frames presents a hard cut, if this difference exceeds a certain threshold. The amount of these values is averaged over a window $\mathrm{N}$ resulting in the hard cut frequency $f_{3}$ (Fig.7). We implemented also one descriptor which builds on the motion-activity information included in the mpeg- 2 stream. 


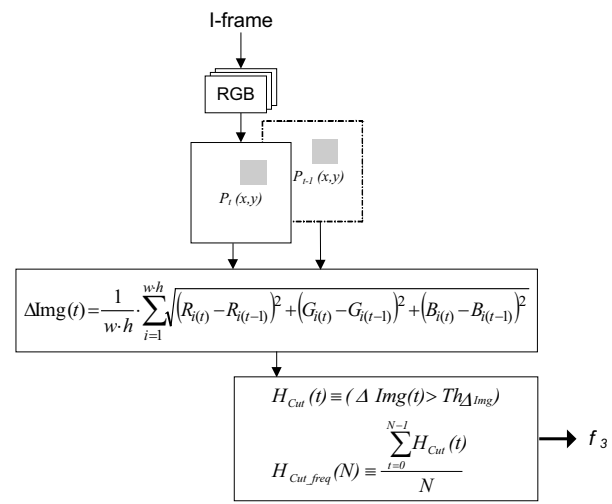

Figure 7: Block diagram of the hard-cut descriptor

We applied the descriptors to the training-data. The extracted features of the commercial sequences appeared within a decision window of 50 I-frames mainly in three combinations, which is shown in Figure 8.

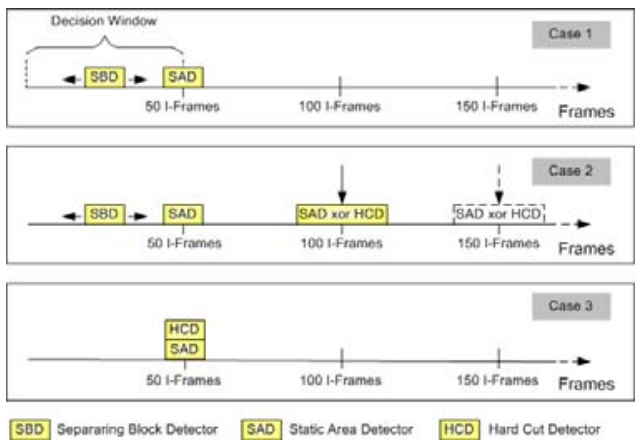

Figure 8: Appearance of features in commercials

In case 1 a separating-block and non-static areas (no-logos) were detected within a decision window.

In case 2 either a high hard-cut frequency or no-logo was detected providing that case 1 occurred within the last $100 \mathrm{I}$ frames. In the third case no-logo and a high hard-cutfrequency were simultaneously detected.

\subsection{Decision-Tree}

These cases are combined with a decision tree according to the ID3 algorithm [10]. We calculate the average entropy $E$ for each case from the training-data shown in Table 1.

$$
E_{\text {average }}=\sum_{j=1}^{b}\left(\left(\frac{n_{b}}{n_{t}}\right) \cdot\left[\sum_{i=1}^{c}-\left(\frac{n_{b c}}{n_{b}}\right) \log _{2}\left(\frac{n_{b c}}{n_{b}}\right)\right]\right)
$$

\begin{tabular}{|l|c|c|c|c|c|c|}
\hline & \multicolumn{3}{|c|}{ Commercial } & \multicolumn{3}{c|}{ Hon-Commercial } \\
\hline & $\begin{array}{c}\text { correct } \\
\text { accept. }\end{array}$ & $\begin{array}{c}\text { accept. } \\
\text { aclse }\end{array}$ & $\Sigma$ & $\begin{array}{c}\text { correct } \\
\text { reject. }\end{array}$ & accept. & $\Sigma$ \\
\hline case 1 & 82 & 0 & $\mathbf{8 2}$ & 16 & 384 & $\mathbf{4 0 0}$ \\
\hline case 2 & 8 & 0 & $\mathbf{8}$ & 90 & 348 & $\mathbf{4 7 4}$ \\
\hline case 3 & 56 & 2 & $\mathbf{5 8}$ & 42 & 382 & $\mathbf{4 2 4}$ \\
\hline
\end{tabular}

Table 1: Cases with accepted/rejected rate as commercial
The case with the lowest entropy forms the first node. The resulting decision tree is shown in Figure 9.

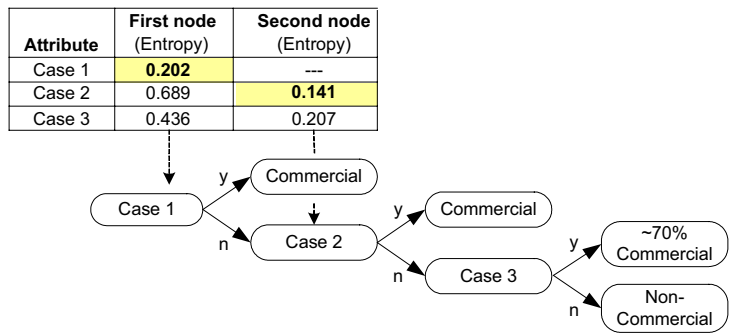

Figure 9: Block diagram of the developed decision tree

\section{EXPERIMENTS}

The experiments were carried out on a database of 200 representative video sequences (100 sequences for training and 100 as testing-data), in total of $400 \mathrm{~min}$ of recordings; 40 'commercials' and 4*40 'non-commercials' (cartoon, music, news and sport) of 2 minutes' each gathered from popular TV in Germany (ARD, ZDF, BBC, VIVA..). We extracted frames and scaled them down to a resolution of $90 * 72$ pixels. The number of considered frames in the processing window is $\mathrm{N}=50 \mathrm{I}$-frames.

\subsection{Experimental Results}

We examined the performance of each descriptor on the training-data. The separating-block descriptor should detect the transition from a running spot to a new spot.

\begin{tabular}{lcc} 
Genre & $\begin{array}{c}\text { observed number } \\
\text { of sep. blocks }\end{array}$ & $\begin{array}{c}\text { detected number } \\
\text { of sep. blocks }\end{array}$ \\
\hline Commercial & 111 & 108 \\
Cartoon & 0 & 2 \\
Music & 0 & 7 \\
News & 0 & 0 \\
Sport & 0 & 0
\end{tabular}

Table 2: Classification accuracy of the sep.-block descriptor

From 111 subjectively determined separating blocks within the 20 'commercials' only three blocks, including fades, were misclassified. The same reason caused the false acceptances in 'cartoon' and 'music'. In 'news' and 'sports' the descriptor worked correctly.

Table 3 shows the amount of windows $\mathrm{N}$ within each genre and the detected number of windows with non-static areas.

\begin{tabular}{lcc} 
Genre & $\begin{array}{c}\text { examined number } \\
\text { of windows }\end{array}$ & $\begin{array}{c}\text { detected number of non } \\
\text {-static areas/ no logo }\end{array}$ \\
\hline Commercial & 98 & 90 \\
Cartoon & 94 & 0 \\
Music & 105 & 1 \\
News & 95 & 1 \\
Sports & 90 & 0
\end{tabular}

Table 3: Classification accuracy of the static-area descriptor 
From 98 windows in commercial, 90 with non-static-areas were detected. The remaining 8 contained a static 'company-logo'. In 'music' we had a window including a scene-change without the TV-logo and in 'news' the logo was at the beginning on a window outside the scanned corners.

Table 4 shows the performance of the hard cut descriptor.

\begin{tabular}{lcc} 
Genre & $\begin{array}{c}\text { examined number } \\
\text { of windows }\end{array}$ & $\begin{array}{c}\text { detected num. of windows } \\
\text { with high-cut rate }\end{array}$ \\
\hline Commercial & 98 & 59 \\
Cartoon & 94 & 20 \\
Music & 105 & 45 \\
News & 95 & 4 \\
Sports & 90 & 15
\end{tabular}

Table 4: Classification accuracy of the hard-cut descriptor

The recognition of 'commercials' in a processing window using a single descriptor is obviously not sufficient. In order to achieve high identification rates, we developed the tree with nodes based on logical combinations of the descriptors and a branching ratio of 2 .

Tab. 5 shows the detection rates for the 20 video sequences of each genre used in our experiments.

\begin{tabular}{lcc|c} 
Genre & $\begin{array}{c}\text { examined number } \\
\text { of windows }\end{array}$ & $\begin{array}{c}\text { detected number } \\
\text { of commercials }\end{array}$ & \multicolumn{1}{l}{$(\%)$} \\
\hline Commercial & $\mathbf{9 8}$ & $\mathbf{9 1}$ & $\mathbf{9 3 \%}$ \\
Cartoon & 94 & 0 & $0 \%$ \\
Music & 105 & 1 & $1 \%$ \\
News & 95 & 1 & $1 \%$ \\
Sports & 90 & 0 & $0 \%$ \\
& & &
\end{tabular}

Table 5: Probability for video being detected as Commercial

In our experiment 91 from 98 examined windows from the genre 'commercial' were classified as commercial.

The remaining 7 windows were very close to the commercial-detection threshold of $50 \%$. Those sequences started within a commercial, after the appearance of a separating block, presented a long spot with a 'companylogo' and had a low high-cut-frequency. It is obvious, that detection of sequences with 'special cases' is highly unreliable.

It is interesting to note that only 1 from 105 examined windows of the genre 'music' and 1 of 95 windows of 'news' were misclassified (caused by non-static areas and high cut frequency). The genre 'cartoon' and 'sport' were correctly classified in more than 90 windows.

\section{SUMMARY \& CONCLUSION}

We presented a new approach for the detection of commercials. Three key contributions have been made. We started with new or modified visual descriptors. Second, we considered the temporal relations of the features. Third, we developed a decision tree to combine the results of the visual descriptors, deriving a probability rate for a video sequence being a 'commercial' or 'non-commercial'.
A video database containing five popular genres namely cartoon, commercial, music, news and sports has been used. An average correct classification rate of $93 \%$ for commercial-videos detected as a 'commercial' and more than $99 \%$ for the other genres detected as a "noncommercial' has been achieved.

With our current software system we achieved on an AMD Athlon XP1600+, $1.41 \mathrm{GHz}$ a run-time performance of approximately $1 \mathrm{~min}$ for classification for $1 \mathrm{~min}$ of video.

\section{REFERENCES}

[1] A. Albiol, M.J.C. Fulla, F.A. Albiol and L. Torres, "Detection of TV commercials", IEEE International Conference on Acoustics, Speech, and Signal Processing, volume 3, pp. 541 - 544, 2004.

[2] B. Satterwhite and O. Marques, "Automatic detection of TV commercials”, IEEE Potentials, pp. 9 - 12, 2004.

[3] J.H. Yeh, J.C. Chen, J.H. Kuo and J.L. Wu, "TV Commercial Detection in News Program Videos", IEEE International Symposium on Circuits and Systems, pp. 4594 - 4597, 2004.

[4] R.J.M. den Hollander and A. Hanjalic, "Logo recognition in video stills by string matching", International Conference on Image Processing, volume 3, pp. 517 - 20, 2003.

[5] D. A. Sadlier, S. Marlow, N. O'Connor and N. Murphy, "Automatic TV Advertisement Detection from MPEG Bitstream", International Conference on Enterprise Information Systems, pp. 449 - 452, 2001.

[6] R. Lienhart, C. Kuhmünch and W. Effelsberg, "On the Detection and Recognition of Television Commercials", IEEE Conference on Multimedia Computing and Systems, pp. 509 - 516, 1997.

[7] R. Glasberg, K. Elazouzi and T. Sikora, "Video-genreclassification: recognizing cartoons in real-time using visualdescriptors and a multilayer-percetpron", The 7th International Conference on Advanced Communication Technology, volume 2, pp. $1121-1124,2005$.

[8] M. Roach, J. Mason and L.-Q. Xu, "Video genre verification using both acoustic and visual modes", International Workshop on Multimedia Signal Processing, pp. 157 - 160, 2002.

[9] B. T. Truong, S. Venkatesh and C. Dorai, "Automatic genre identification for content-based video categorization", Proceedings 15th International Conference on Pattern Recognition, volume 4, pp. $230-233,2000$.

[10] J.R. Quinlan, "Decision trees and decision-making", IEEE Transactions on Systems, Man and Cybernetics, volume 20, pp. 339 - 346, 1990.

[11] T. Sikora, P. Salembier and B.S. Manjunath, Introduction to MPEG-7: Multimedia Content Description Interface, John Wiley LTD, ISBN 0471486787, 2002. 